\title{
Coronal abundances of X-ray bright pre-main sequence stars in the Taurus molecular cloud
}

\author{
L. Scelsi ${ }^{1,2}$, A. Maggio ${ }^{2}$, G. Micela ${ }^{2}$, K. Briggs ${ }^{3}$, and M. Güdel ${ }^{3}$ \\ 1 Dipartimento di Scienze Fisiche ed Astronomiche, Sezione di Astronomia, Università di Palermo, Piazza del Parlamento 1, \\ 90134 Palermo, Italy \\ e-mail: scelsi@oapa.astropa.unipa.it \\ 2 INAF - Osservatorio Astronomico di Palermo, Piazza del Parlamento 1, 90134 Palermo, Italy \\ ${ }_{3}$ Paul Scherrer Institut, Würenlingen and Villigen, 5232 Villigen PSI, Switzerland
}

Received 4 May 2007 / Accepted 5 July 2007

\begin{abstract}
Aims. We studied the thermal properties and chemical composition of the X-ray emitting plasma of a sample of bright members of the Taurus Molecular Cloud to investigate possible differences among classical and weak-lined T Tauri stars, and possible dependences of the abundances on the stellar activity level and/or on the presence of accretion/circumstellar material.

Methods. We used medium-resolution X-ray spectra obtained with the sensitive EPIC/PN camera in order to analyze the possible sample. The PN spectra of 20 bright $\left(L_{X} \sim 10^{30}-10^{31} \mathrm{erg} \mathrm{s}^{-1}\right)$ Taurus members, with at least $\sim 4500$ counts, were fitted using thermal models of optically thin plasma with two components and variable abundances of $\mathrm{O}, \mathrm{Ne}, \mathrm{Mg}, \mathrm{Si}, \mathrm{S}, \mathrm{Ar}, \mathrm{Ca}$, and $\mathrm{Fe}$. Extensive preliminary investigations were employed to study the performances of the PN detectors regarding abundance determinations, and finally to check the results of the fittings.

Results. We found that the observed X-ray emission of the studied stars can be attributed to coronal plasma having similar thermal properties and chemical composition both in the classical and in the weak-lined $\mathrm{T}$ Tauri stars. The results of the fittings did not reveal a correlation between the abundance patterns and activity or accretion/disk presence. The iron abundance of these active stars is significantly lower than the solar photospheric value ( 0.2 solar). An indication of slightly different coronal properties in stars with different spectral type is found in this study. G-type and early K-type stars have, on average, slightly higher Fe abundances (Fe $\sim 0.24$ solar) than stars with later spectral type (Fe $\sim 0.15$ solar), confirming previous findings from high-resolution X-ray spectroscopy. Stars of the former group are also found to have, on average, hotter coronae.
\end{abstract}

Key words. X-rays: stars - stars: activity - open clusters and associations: individual: name: Taurus molecular cloud - stars: abundances - stars: coronae - stars: pre-main-sequence

\section{Introduction}

Studies on the chemical composition of stellar atmospheres are important to address several issues in astrophysics, such as the evolution of stellar galactic populations, the enrichment of the interstellar medium, the influence of elemental abundances on star formation processes, and on the structure of stellar interiors through opacity effects. In the framework of coronal physics, solar and stellar observations in the UV and X-ray bands during the last two decades have suggested abundance anomalies that, if understood, could provide powerful new diagnostics for the physics of stellar outer atmospheres. In particular, previous observations of the Sun (Meyer 1985; Feldman 1992), showed different chemical abundances in corona with respect to the photosphere, with a pattern depending on the first ionization potential (FIP) of the various elements. The present view is that elements with FIP $\lesssim 10 \mathrm{eV}$ (low-FIP elements $\mathrm{Mg}, \mathrm{Si}, \mathrm{Ca}, \mathrm{Ni}, \mathrm{Fe}$ ) are overabundant in the solar corona with respect to the photosphere by a factor of $\sim 4-5$; elements with FIP $\gtrsim 10 \mathrm{eV}$ (highFIP elements $\mathrm{C}, \mathrm{S}, \mathrm{O}, \mathrm{Ne}, \mathrm{Ar}$, with $\mathrm{C}$ and $\mathrm{S}$ lying close to the boundary of this definition) have coronal abundances similar to the corresponding photospheric values. This solar-type FIP effect is most clearly present in long-lived active regions, while it is much less marked (or even absent) in coronal holes (Widing \& Feldman 1992; Sheeley 1996). In order to compare solar and spatially-integrated stellar observations, Laming et al. (1994) investigated full-disk solar spectra in the extreme ultraviolet band (EUV), and detected the FIP effect also in this case.

Analyses of EUV line emission spectra from the coronae of low- and intermediate-activity stars ( $\alpha$ Centauri, $\xi$ Bootis, $\epsilon$ Eridani, observed with the EUVE satellite) revealed solar-like abundance anomalies, suggesting that the FIP effect is not an isolated solar phenomenon (Laming et al. 1996; Drake et al. 1997; Laming \& Drake 1999). New studies, based on XMM-Newton and Chandra high-resolution X-ray spectra, have led to claims of different FIP-dependent abundance patterns in the coronae of other stars: in particular, observations of very active stars, both single stars and active binary systems, suggested a dependence of coronal abundances on FIP that is reversed with respect to the Sun and the other low-activity stars mentioned above, i.e. a depletion of low-FIP elements with respect to high-FIP elements (inverse FIP effect; Brinkman et al. 2001; Audard et al. 2003; Robrade \& Schmitt 2005). Telleschi et al. (2005) derived coronal abundances for 6 solar analogs (i.e. G-type main sequence stars) with different ages and rotation periods, and hence activity levels: this work suggested a change from an inverse FIP effect to a solar-type FIP effect with increasing age, and hence with decreasing activity.

In contrast, other authors found evidence for neither a FIP nor an inverse FIP effect in the coronae of active late-type stars 
(Sanz-Forcada et al. 2003; Argiroffi et al. 2004; Scelsi et al. 2005), but instead a minimum in the abundance pattern occurring at FIP $\sim 10 \mathrm{eV}$, with increasing abundances at both lower and higher FIP values. In any case, a systematic result emerging from stellar abundance studies is that the coronae of highactivity stars appear significantly metal-poor relative to the solar photosphere, with typical iron abundances $\sim 0.1-0.3$ times the Anders \& Grevesse (1989) solar photospheric value.

A major problem encountered in coronal abundance studies is related to data analysis issues. The requirements for an accurate analysis are good quality X-ray spectra (i.e. high $S / N$ ratios), a careful selection of emission lines (not affected by blends and independent from the plasma density), accurate line emissivity functions describing the physics of atomic transitions, and a precise knowledge of the instrumental response. All these difficulties introduce errors that are often not accounted for in the reported (statistical) measurement uncertainties, which therefore are likely underestimated in many studies.

While the coronal metal deficiency of active stars seems to be a rather solid result, the question whether coronal abundances are different from the photospheric values, with a pattern possibly dependent on the FIP, is still debated and deserves further investigation, especially because photospheric abundances of active, rapid rotators are mostly unavailable. Better observational evidence is definitely required to test theoretical models proposed to explain the physical mechanism(s) responsible for such differences (e.g. Schwadron et al. 1999; Laming 2004).

In classical T Tauri stars (CTTSs) material from the circumstellar disk accretes onto the stellar surface and shock waves at the base of the accretion funnel are expected to heat the gas to X-ray emitting temperatures of a few million degrees. From recent analyses of XMM-Newton and Chandra spectra of premain sequence stars, interesting peculiarities in the abundances of some CTTSs were also noted: TW Hydrae, TWA 5, and BP Tauri show anomalous $\mathrm{Ne} / \mathrm{Fe}$ abundance ratios $\sim 5-10$ solar (Stelzer \& Schmitt 2004; Argiroffi et al. 2005; Robrade \& Schmitt 2006), significantly higher than those typically found for other stars $(A(\mathrm{Ne}) / A(\mathrm{Fe}) \sim 3)$. Such peculiarities could be inherited from the original cloud where these stars formed, but they might also be due to some process of chemical segregation during the evolution of circumstellar disks. In fact, most of the soft X-ray emission of TW Hydrae and part of the emission of BP Tauri have been attributed to accretion shocks, while the situation for TWA 5 is more uncertain. Iron and other metals in the disk could condense in grains, as predicted by some models of cloud chemistry (Savage \& Sembach 1996; Charnley 1997). If we suppose that the gas and dust phases in the circumstellar disk are separated for some reason, with the metals remaining at larger distance from the star, while neon and other noble elements in the gas phase accrete more efficiently onto the stellar surface, then the gas heated by the shock at X-ray emitting temperatures is expected to be metal-deficient and enriched in neon. The high Ne/O ratio of TW Hydrae may be another sign of such a segregation process occurring in the circumstellar disk of this star, as argued by Drake et al. (2005). Should this scenario be proven correct for the CTTSs and the gas-dust separation mechanism understood, the chemical composition of the hot plasma could provide new insight into the study of circumstellar disks. The relevance for our understanding of planetary formation could also be significant.

Most recently, Maggio et al. (2007) studied a sample of $146 \mathrm{X}$-ray bright sources associated with members of the young Orion Nebula Cluster (ONC), and found that their emission originates from a plasma with temperatures and elemental abundances very similar to those of active coronae in older stars. Moreover, the abundance distributions are compatible with a single pattern vs. FIP for all elements in all stars with the only possible exception being the calcium abundance. Comparison with abundances in stellar photospheres and in the gaseous component of the nebula indicate a significant underabundance at coronal level only for iron (by a factor 1.5-3), while there is agreement (and hence no evidence of a FIP-related effect) for all the other elements with available measurements.

In another recent study based on nine high-resolution RGS spectra from the XEST survey, Telleschi et al. (2007) found similar abundances in accreting and non-accreting stars and indication of higher Fe abundances in the G-type stars of their sample compared to stars with later spectral type. They also found that CTTSs display a soft excess when compared to weak-lined T Tauri stars (WTTSs) or zero-age main-sequence stars, and that high electron densities and $\mathrm{Ne} / \mathrm{Fe}$ abundance ratios are not characteristic of all accreting pre-main sequence (PMS) stars.

In this paper we analyze X-ray spectra taken with $\mathrm{XMM}-$ Newton to derive the thermal properties and chemical composition of the X-ray emitting plasma of a sample of 20 young stellar objects (7 CTTSs and 13 WTTSs) belonging to the Taurus-Auriga star forming region and spanning an age range of $\sim 1-10$ Myr. This region has recently been the subject of the XMM-Newton Extended Survey of Taurus (XEST, Güdel et al. 2007), which has allowed us to study the X-ray emission from more than 150 classical and weak-lined $\mathrm{T}$ Tauri stars in the Taurus Molecular Cloud (TMC). The present work focuses on some of the most active members of the TMC, with the aim to understand whether accreting and non-accreting pre-main sequence stars show any systematic difference in the abundance pattern, and to investigate possible dependences of the coronal abundances on the activity level.

The paper is organized as follows. Section 2 summarizes relevant information about the TMC and the XEST observations. In Sect. 3 we describe the strategy employed to select the sample and we report the spectral fitting results, whose discussion is presented in Sect. 4. Finally, we draw our conclusions in Sect. 5. Appendix A details the simulations performed for this work.

\section{The TMC and the XEST observations}

The TMC is a cloud complex at $140 \mathrm{pc}$ from the Sun, which contains about 400 known low-mass members; they are spread over a relatively large portion of the sky ( $\sim 100$ square degrees $)$ and their ages cover a range between $10^{5}$ and $3 \times 10^{7}$ years, with most stars being typically 1-10 million years old. The XEST survey consists of 19 recent observations (fields XEST-02 to XEST-20), obtained in two separate periods (August-September 2004 and February-March 2005) and with exposures ranging from 27 to $35 \mathrm{ks}$, to which 8 more previous fields ${ }^{1}$ from the XMM-Newton archive were added, having longer exposure times in the range $43-131 \mathrm{ks}$. In total, the surveyed area covers only $\sim 5$ square degrees of the TMC; however, since the observed fields were chosen so as to include the densest regions of the TMC with the largest concentrations of stars, the total number of detections associated to Taurus members in the EPIC images is 159. Including non-resolved companions that might be contributing to the X-ray emission, the XEST survey likely detected around 210 members, that is about a half of the entire known stellar population in Taurus. This sample contains mainly classical and weak-lined

1 The field L1495 around V410 Tau was observed twice; the two observations are labelled XEST-23 and XEST-24. 
T Tauri stars, but also protostars, brown dwarfs, a few Herbig $\mathrm{Ae} / \mathrm{Be}$ stars, and other members with uncertain classification.

The present study is based on X-ray spectra taken with the most sensitive instrument onboard XMM-Newton, the EPIC PN camera (Strüder et al. 2001). This non-dispersive CCD detector has an effective area of $\sim 1200 \mathrm{~cm}^{2}$ at $1.5 \mathrm{keV}$ and provides moderate spectral resolution $R=E / \Delta E \sim 5-50$ in the range $0.1-10 \mathrm{keV}$. Bright sources in the field XEST-26 (around SU Aur), which lacks PN data, were excluded from the analysis to ensure homogeneity of the results. For the XEST observations, the PN was operated in full window mode and the medium filter was applied in all observations, with the exception of the field XEST-27 (around $\zeta$ Per) where the thick filter was used.

We refer to the paper by Güdel et al. (2007) for more details about all the observations of the XEST project, which were processed using SAS v6.1, and the data reduction steps.

\section{Data analysis}

\subsection{Preliminary simulations and choice of the sample}

A study of the chemical composition and of possible effects due to activity or accretion processes requires a large sample of stars and accurate and precise abundance measurements. Accurate estimates of abundances can be derived with the analysis of highresolution spectra, where individual emission lines of several chemical elements are well resolved. The XEST survey has provided useful RGS spectra for nine TMC members, which have been analyzed by Telleschi et al. (2007).

In order to consider a larger sample of T Tauri stars, we based our study on the medium resolution spectra taken with the PN camera, which is $\sim 10$ times more sensitive than the RGS spectrometers and, unlike the latter, can also provide data for sources located off-axis in the field-of-view. The use of PN data required a preliminary feasibility study to understand the reliability of the results obtained from their analysis. In fact, the blending of the lines of many elements (neon, iron, nickel, oxygen) and the method used to analyze these spectra (i.e. global fitting with a plasma emission model with few isothermal components) might lead to systematic uncertainties in the abundances derived for some of the elements. In particular, we addressed two issues: (i) is the global fitting of PN spectra able to recover the true values of elemental abundances, considering sources in a variety of physical conditions and (ii) what minimum number of detected source counts are required to obtain measurements with relatively low abundance uncertainties, within a factor of 2 .

These two issues are clearly not unrelated, since the ability of deriving an accurate and precise abundance value for a given element depends on a) whether the lines of that element have a good signal, and hence on the element abundance, on the thermal structure of the plasma, and also on the amount of interstellar absorption, and b) the statistics of the entire spectrum, which must allow a good determination of the source thermal properties. We also point out that facing these issues requires the most accurate knowledge of the instrumental response function allowed by the instrument calibration.

To answer the above questions, we performed a large number of simulations exploring a variety of source conditions. At the same time, this procedure allowed us to put a rough threshold on the total number of source counts, below which the fitting parameters begin to be poorly constrained, and hence to define the sample of stars to be studied. The approach is analogous to that employed by Maggio et al. (2007) for their abundance study on a sample of stars in Orion, observed with Chandra/ACIS.
Table 1. Sample of TMC members selected for the abundance study. "XEST id" is the identification label in the XEST catalog, the first two digits referring to the XEST field. For each source, both the total exposure time of the relevant observation and the screened time (GTI) selected for this study are reported; "PN cts" are the source counts during the GTIs. "W" and "C" stand for WTTS and CTTS, respectively. TTS types and spectral types are taken from Güdel et al. (2007) and references therein, except where noted.

\begin{tabular}{|c|c|c|c|c|c|c|}
\hline Name & $\overline{\text { XEST id }}$ & $\begin{array}{l}\text { Total } \\
\text { Exp. } \\
\text { (ks) }\end{array}$ & $\begin{array}{l}\text { GTI } \\
(\mathrm{ks})\end{array}$ & $\begin{array}{l}\overline{\mathrm{PN}} \\
\text { cts }\end{array}$ & $\begin{array}{l}\text { TTS } \\
\text { type }\end{array}$ & $\begin{array}{l}\text { Spec } \\
\text { type }\end{array}$ \\
\hline Anon 1 & $20-005$ & 26.8 & 26.8 & 4520 & $\overline{\mathrm{W}}$ & M0 \\
\hline CoKu Tau 3 & $12-059$ & 26.4 & 25.7 & 10454 & W & M1 \\
\hline DN Tau & $12-040$ & 26.4 & 25.7 & 5169 & $\mathrm{C}$ & M0 \\
\hline IT Tau & $18-030$ & 26.8 & 23.8 & 8605 & $\mathrm{C}$ & $\mathrm{K} 2$ \\
\hline L1551-51 & 22-089 & 48.9 & 45.4 & 4506 & W & K7 \\
\hline V773 Tau & $20-042$ & 26.8 & 26.8 & 29971 & W & $\mathrm{K} 2$ \\
\hline V826 Tau & $22-100$ & 48.9 & 45.4 & 16950 & W & K7 \\
\hline${\mathrm{V} 830 \mathrm{Tau}^{a}}^{a}$ & 04-016 & 27.6 & 26.1 & 8765 & W & K7 \\
\hline $\mathrm{DH} \mathrm{Tau}^{a}$ & $15-040$ & 27.4 & 23.3 & 14780 & $\mathrm{C}$ & M1 \\
\hline HD 283572 & 21-039 & 41.1 & 33.9 & 79424 & W & G5 \\
\hline HP Tau/G2 & 08-051 & 33.7 & 31.2 & 15033 & W & G0 \\
\hline Hubble 4 & $23-056$ & 62.6 & 51.9 & 16394 & W & K7 \\
\hline KPNO-Tau 15 & 08-043 & 33.7 & 31.2 & 6017 & W & M2.75 \\
\hline RY Tau & $21-038$ & 41.1 & 33.9 & 5685 & $\mathrm{C}$ & K1 \\
\hline V410 Tau & $24-028$ & 36.9 & 27.4 & 26740 & W & K4 \\
\hline V819 Tau & 23-074 & 62.6 & 51.9 & 5524 & $\mathrm{~W}$ & K7 \\
\hline V892 Tau & 23-047 & 62.6 & 51.9 & 10886 & $\mathrm{C}^{c}$ & $\mathrm{~K} 2 ?^{c}$ \\
\hline BP Tau ${ }^{b}$ & $28-100$ & 116.4 & 88.0 & 17770 & $\mathrm{C}$ & K7 \\
\hline $\mathrm{T} \mathrm{Tau}^{a}$ & 01-045 & 67.2 & 37.5 & 29960 & $\mathrm{C}$ & K0 \\
\hline RXJ0422.1+1934 & 01-054 & 67.2 & 37.5 & 11296 & $\mathrm{~W}$ & - \\
\hline
\end{tabular}

${ }^{a}$ The light curve suggests that the emission is affected by the final stage of a flare decay. ${ }^{b}$ A flare occurred during the observation is excluded from the GTIs. ${ }^{c}$ Secondary of the binary system.

In brief, our simulations, whose details are reported in Appendix A, showed that the abundances of most elements can be rather accurately derived from fitting of PN spectra. An indicative threshold of $\sim 5000$ total counts was set in order to have useful PN data for abundance studies. Uncertainties at $90 \%$ level are usually better by a factor of $\sim 2.5$ for spectra with $\sim 5000$ counts, and better by a factor of $\sim 2$ for spectra with $\sim 10000$ counts. The iron abundance is the best constrained in most simulations, although some caveats must be noted in a few cases (see Appendix A). Instead, the abundances of Ar and $\mathrm{Ca}$ are generally very poorly constrained, as well as the $\mathrm{O}$ abundance in case of high absorption $\left(N_{\mathrm{H}}>10^{21} \mathrm{~cm}^{-2}\right)$.

Therefore, we selected all the TMC members with more than 5000 counts in the PN spectra $(0.3-10 \mathrm{keV})$, integrated over time intervals not affected by high background levels. We also added to the sample the two stars Anon 1 and L1551-51 with $\sim 4500$ counts. The sample defined in this way is listed in Table 1 and consists of 20 TMC members, 13 of which are weak-lined T Tauri stars, and the remaining 7 are accreting PMS stars (1 Herbig Be star and 6 CTTSs). Note that the Herbig Be star in this sample, V892 Tauri, has a close companion $\left(0.05^{\prime \prime}\right.$ separation) with an estimated mass of $1.5-2 M_{\odot}$ (Smith et al. 2005), which therefore is likely to be the main source of Xray emission, rather than the Herbig Be star itself. It is also likely that such a companion is an accreting star, based on previous studies (Duchêne et al. 1999; König et al. 2001; Hartigan \& Kenyon 2003) showing that mixed binary systems, i.e. systems that include both accreting and non-accreting stellar components, are very uncommon. Using the evolutionary models by 
Table 2. Parameters of the best-fit models for the $20 \mathrm{TMC}$ members, with $1 \sigma$ errors. The unabsorbed X-ray luminosity $L_{\mathrm{X}}$ and the surface flux $F_{\mathrm{X}}$ are calculated in the $0.3-10 \mathrm{keV}$ band. $T_{\mathrm{av}}$ is defined as $T_{\mathrm{av}}=\left(E M_{1} T_{1}+E M_{2} T_{2}\right) /\left(E M_{1}+E M_{2}\right)$.

\begin{tabular}{|c|c|c|c|c|c|c|c|c|}
\hline Name & $\begin{array}{c}N_{\mathrm{H}} \\
\left(10^{21} \mathrm{~cm}^{-2}\right)\end{array}$ & $\begin{array}{c}T_{1} \\
(\mathrm{MK})\end{array}$ & $\begin{array}{c}T_{2} \\
(\mathrm{MK})\end{array}$ & $\overline{E M_{2} / E M_{1}}$ & $\chi_{v} 2$ (d.o.f.) & $\begin{array}{c}L_{\mathrm{X}} \\
\left(10^{30} \mathrm{erg} \mathrm{s}^{-1}\right)\end{array}$ & $\begin{array}{c}T_{\mathrm{av}} \\
(\mathrm{MK})\end{array}$ & $\begin{array}{c}F_{\mathrm{X}} \\
\left(10^{6} \mathrm{erg} \mathrm{s}^{-1} \mathrm{~cm}^{-2}\right)\end{array}$ \\
\hline Anon 1 & $4.9 \pm 0.7$ & $4.8_{-0.6}^{+1.1}$ & $29_{-8}^{+22}$ & $0.12 \pm 0.06$ & $0.96(141)$ & $8.1_{-22}^{+5}$ & 7.4 & 10.0 \\
\hline CoKu Tau 3 & $4.6 \pm 0.4$ & $4.3 \pm 0.7$ & $18.6 \pm 1.2$ & $1.2 \pm 0.4$ & $0.88(284)$ & $7.4_{-0.9}^{+2.2}$ & 12.2 & 20.6 \\
\hline DN Tau & $0.44 \pm 0.20$ & $8.7 \pm 0.8$ & $24 \pm 3$ & $1.6 \pm 0.8$ & $1.18(142)$ & $1.10_{-0.05}^{+0.12}$ & 18.4 & 3.4 \\
\hline IT Tau & $7.0_{-0.6}^{+1.2}$ & $10_{-2}^{+3}$ & $35 \pm 5$ & $4.4 \pm 3$ & $0.92(256)$ & $6.6_{-05}^{+1.8}$ & 30.7 & 22.8 \\
\hline L1551-51 & $0.78 \pm 0.23$ & $7.4 \pm 0.7$ & $21_{-4}^{+10}$ & $0.6 \pm 0.3$ & $1.04(128)$ & $1.75_{-0.09}^{+0.35}$ & 12.3 & 14.0 \\
\hline V773 Tau & $1.58 \pm 0.10$ & $4.8_{-0.3}^{+0.5}$ & $24.3^{-4} \pm 1.3$ & $2.0 \pm 0.3$ & $1.07(434)$ & $9.0_{-0.6}^{-1.099}$ & 17.9 & 40.2 \\
\hline V826 Tau & $1.03 \pm 0.11$ & $4.7_{-0.4}^{+0.8}$ & $17.9 \pm 1.6$ & $0.89 \pm 0.22$ & $1.16(304)$ & $5.1 \pm 0.3$ & 11.0 & 21.6 \\
\hline V830 Tau & $0.79 \pm 0.20$ & $6.8_{-2.0}^{+1.4}$ & $23 \pm 6$ & $0.9 \pm 0.4$ & $1.17(237)$ & $5.7_{-0.4}^{+1.0}$ & 14.5 & 28.7 \\
\hline DH Tau & $1.82 \pm 0.20$ & $8.9 \pm 0.5$ & $25 \pm 3$ & $1.1 \pm 0.4$ & $1.04(319)$ & $7.7_{-0.9}^{-0.4}$ & 17.3 & 37.7 \\
\hline HD 283572 & $0.55 \pm 0.05$ & $8.5 \pm 0.2$ & $24.5 \pm 1.1$ & $1.96 \pm 0.21$ & $1.02(599)$ & $12.4^{-0.4} \pm 0.3$ & 19.1 & 30.7 \\
\hline HP Tau/G2 & $3.6 \pm 0.3$ & $8.7 \pm 0.5$ & $22.8 \pm 1.4$ & $1.9 \pm 0.5$ & $0.94(329)$ & $8.1_{-05}^{+1.1}$ & 18.0 & 24.1 \\
\hline Hubble 4 & $2.96 \pm 0.21$ & $4.7_{-0.4}^{+0.6}$ & $19.2 \pm 1.7$ & $1.01 \pm 0.21$ & $1.14(341)$ & $6.2_{-0.4}^{+0.9}$ & 12.0 & 9.1 \\
\hline KPNO-Tau 15 & $3.6 \pm 0.5$ & $9.5_{-1.5}^{+2.4}$ & $33 \pm 5$ & $3.2 \pm 2.1$ & $0.95(200)$ & $2.5 \pm 0.3$ & 27.0 & 36.7 \\
\hline RY Tau & $9.3 \pm 2.6$ & $5.3 \pm 1.6$ & $35_{-6}^{+9}$ & $1.0 \pm 0.9$ & $0.79(203)$ & $8_{-3}^{+11}$ & 19.8 & 10.7 \\
\hline V410 Tau & $0.40 \pm 0.07$ & $4.7 \pm 0.4$ & $20.8^{-6} \pm 1.5$ & $1.50 \pm 0.20$ & $1.04(364)$ & $4.93_{-0.12}^{+0.26}$ & 14.4 & 14.9 \\
\hline V819 Tau & $1.6 \pm 0.4$ & $7.2 \pm 0.7$ & $19 \pm 4$ & $0.9 \pm 0.4$ & $0.95(175)$ & $2.3 \pm 0.6$ & 12.6 & 10.0 \\
\hline V892 Tau & $9.2 \pm 0.8$ & $10.8_{-1.6}^{+1.1}$ & $32 \pm 7$ & $0.9 \pm 0.4$ & $0.94(325)$ & $9.0_{-1.2}^{+2.5}$ & 20.8 & 20.7 \\
\hline BP Tau & $0.78 \pm 0.11$ & $4.7 \pm 0.4$ & $22.6 \pm 2.3$ & $1.10 \pm 0.18$ & $0.99(338)$ & $1.45_{-0.07}^{+0.12}$ & 14.1 & 6.0 \\
\hline T Tau & $2.53 \pm 0.16$ & $9.0 \pm 0.4$ & $28.9 \pm 1.9$ & $2.0 \pm 0.6$ & $1.22(513)$ & $7.6_{-0.3}^{+0.01}$ & 22.2 & 9.4 \\
\hline RXJ0422.1+1934 & $3.4 \pm 0.3$ & $4.4 \pm 0.6$ & $19.7 \pm 1.5$ & $1.5 \pm 0.4$ & $0.93(302)$ & $3.7_{-0.3}^{+0.7}$ & 13.6 & - \\
\hline
\end{tabular}

Siess et al. (2000) and age equal to that of the primary star ( $\sim 3 \mathrm{Myr})$, the spectral type of this companion is $\mathrm{K} 3$ for $M=$ $1.5 M_{\odot}, \mathrm{K} 2$ for $M=1.7 M_{\odot}$, and $\mathrm{K} 1$ for $M=2 M_{\odot}$. In the following we will assume a spectral type of K2.

\subsection{Spectral fittings of TMC members}

The PN counts of the selected TMC members were extracted from circular regions around the sources, having typical radii of $\sim 40^{\prime \prime}-50^{\prime \prime}$. For each source, the background events were extracted from an annulus around the source; contamination from close X-ray sources within the annulus was also avoided (see Güdel et al. 2007, for more details about the calculation of the source and background extraction regions). The standard tasks of the Science Analysis Software (SAS) were used to build the instrumental responses for all sources. In Table 1 we report both the total exposure times of the fields where the selected targets lie and the good time intervals obtained by excluding from the present analysis those time intervals affected by high background emission (due to solar events), in order to increase the $S / N$ ratios of the studied spectra at high energies; in the case of BP Tau, a $\sim 30 \mathrm{ks}$ interval when the source exhibited a strong flare was also excluded, so as to avoid possible effects of time variation of the abundances, as suggested by a number of previous studies (Tsuboi et al. 1998; Güdel et al. 1999; Favata \& Schmitt 1999).

All PN spectra were fitted with the software XSPEC, limiting the fitting of each source to those energy bins where the background spectrum does not significantly affect the source emission (usually $0.3-7 \mathrm{keV}$, in very few cases we excluded the energy bins at $E>5 \mathrm{keV}$ ). We used a two-component thermal, optically thin plasma based on the APEC emissivity code, plus interstellar absorption, which is the same model employed for the simulations. The model has 13 free parameters: the hydrogen column density $N_{\mathrm{H}}$, the temperatures $T_{1}$ and $T_{2}$ and the emission measures $E M_{1}$ and $E M_{2}$ of the two components, and the abundances of $\mathrm{O}, \mathrm{Ne}, \mathrm{Fe}, \mathrm{Mg}, \mathrm{Si}, \mathrm{S}, \mathrm{Ca}$ and Ar. For each source, the $N_{\mathrm{H}}$ value, the two temperatures and the ratio of the emission measures of the best-fitting model are reported in Table 2, together with the unabsorbed X-ray luminosity derived from the model, the average temperature and the surface X-ray flux derived from $L_{X}$ and the stellar radius (the latter is taken from the Tables in Güdel et al. 2007). Average temperatures are weighted by the emission measure and are useful to compare the global thermal properties of the emitting plasma in different stars; they are indicative values and are reported without errors in the table. The surface fluxes are also reported without errors, owing to uncertainties in the adopted stellar radii. In Fig. 1 we plot the observed spectra with their relevant model spectra for some example cases.

Figure 2 shows the properties of the X-ray emitting plasma of the studied sample. The distributions of temperatures and EM ratios are very similar in the subsamples of WTTSs and CTTSs; the latter seems to have, on the whole, average temperatures slightly greater than the WTTS (the medians of the distributions of $T_{\mathrm{av}}$ are $13.5 \mathrm{MK}$ and $19.5 \mathrm{MK}$, respectively), although a Kolmogorov-Smirnov test gives $7 \%$ probability that the distributions for WTTSs and CTTSs are not distinguishable.

In Fig. 3 we plot the derived abundances (in solar units of Anders \& Grevesse 1989) of the TMC members, where the elements are ordered by increasing FIP. The 20 values of abundances are rather widely distributed. The medians of their distributions show a pattern with a minimum around $\mathrm{Fe}$ and $\mathrm{Si}$, a trend already obtained from the analysis of high-resolution spectra for several main sequence, PMS and giant stars with high X-ray luminosity (Sanz-Forcada et al. 2003; Argiroffi et al. 2004; Scelsi et al. 2004; Argiroffi et al. 2005; Scelsi et al. 2005; Telleschi et al. 2005). However, as mentioned above, the Ca abundance was generally ill-constrained in the simulations, which showed that nearly solar values can be frequently obtained even when the input $\mathrm{Ca}$ abundance is significantly subsolar; hence, if we do 

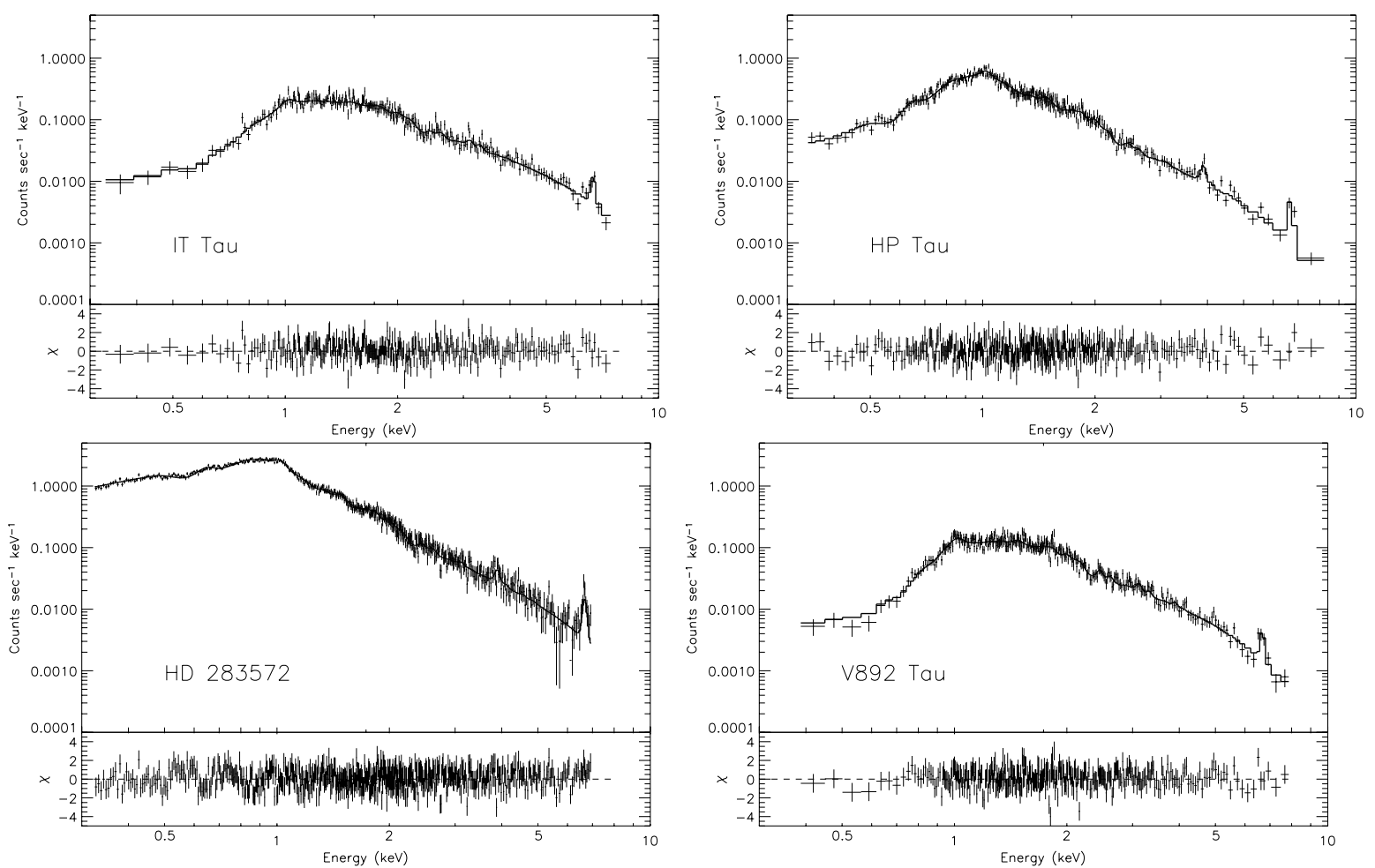

Fig. 1. Examples of PN spectra (points with error bars) and best-fit model spectra (histogram) of selected TMC members in the present study. The bottom of each plot shows the residuals in units of $\chi$.

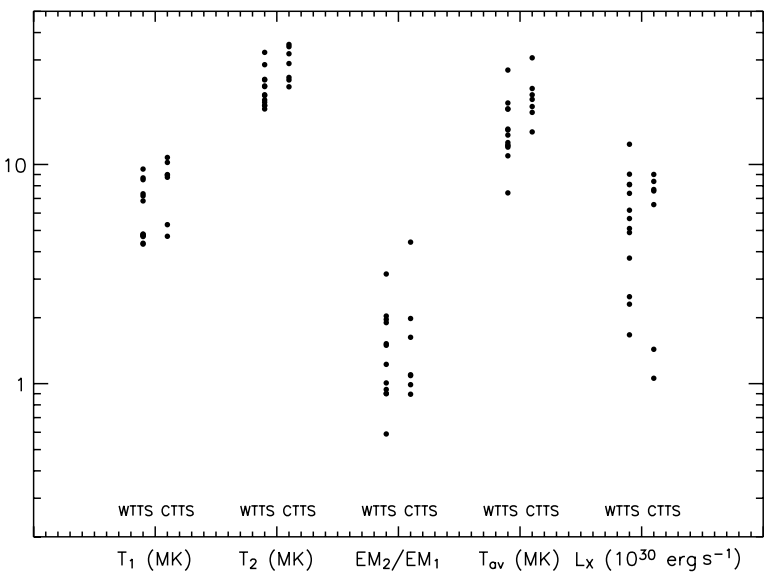

Fig. 2. Temperature, emission measure and X-ray luminosity values for the WTTSs and the CTTSs of the studied sample. The values on the $y$ axis have different units for each quantity, as reported in the $x$-axis labels.

not consider calcium, the pattern of the medians resembles an inverse FIP effect.

It is important to note, however, that the medians of the abundance distributions express average properties of the studied sample, while the abundances of individual stars might be correlated with other stellar parameters. Yet, comparison of Fig. 3 with the results of the simulations shows that the spread of the derived abundances for a given element is roughly of the same magnitude as the one found from the simulations (when comparing Fig. 3 and Fig. A.1 we must consider that half of our sample stars have less than $\sim 10000$ counts). This implies that with the available data we can hardly distinguish between the

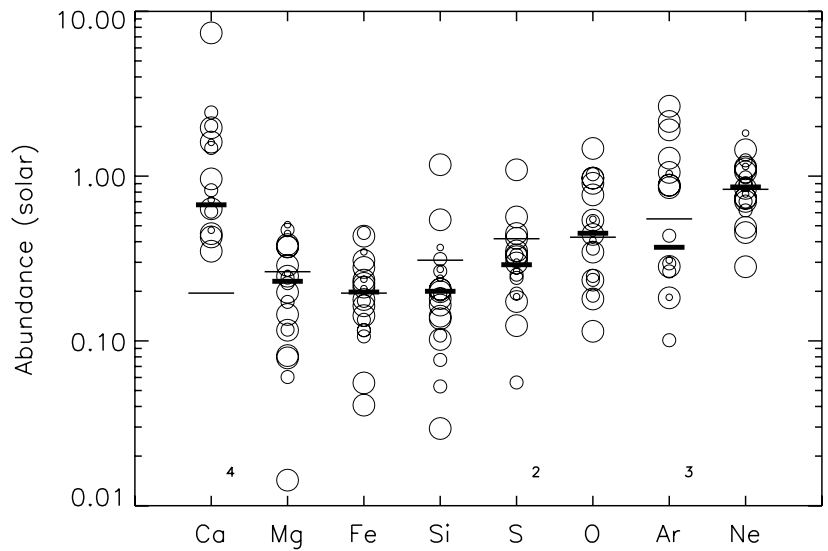

Fig. 3. Abundances of the $20 \mathrm{TMC}$ members for each element, ordered by increasing FIP. The size of the circles gives a rough indication of the accuracy of the measurements: large circles for abundances derived from spectra with less than 10000 counts, medium circles for $10000<$ counts $<20000$, and small circles for counts $>20000$. At the bottom of the plot, close to the label of each element, the number of stars with abundances lying outside the plot (i.e. lower than 0.01 solar) are indicated. The short thick segments mark the medians of the abundance distributions, while the long thin segments mark the values assumed by Güdel et al. (2007) to fit EPIC spectra of Taurus members (see Sect. 4.1).

abundances of any given element in different stars of the present sample, and hence it is not meaningful to search for possible correlations with other parameters. This result also suggests that the derived sets of abundances of these TMC members could be drawn from a single set of abundances, i.e. the spread of the values for any given element is not due to differences among the sources, but rather to statistical fluctuations. To test this 
Table 3. Medians of the distributions of absorption, temperatures, emission measures and abundances derived from the PN spectra of the studied sample. Abundances are in solar units, $N_{\mathrm{H}}$ in $10^{21} \mathrm{~cm}^{-2}, T_{1}$ and $T_{2}$ in MK.

\begin{tabular}{cccccccccccc}
\hline \hline$N_{\mathrm{H}}$ & $T_{1}$ & $T_{2}$ & $E M_{2} / E M_{1}$ & $\mathrm{Ca}$ & $\mathrm{Mg}$ & $\mathrm{Fe}$ & $\mathrm{Si}$ & $\mathrm{S}$ & $\mathrm{O}$ & $\mathrm{Ar}$ & $\mathrm{Ne}$ \\
\hline 2 & 7 & 23 & 1.2 & 0.67 & 0.23 & 0.195 & 0.20 & 0.29 & 0.45 & 0.37 & 0.86 \\
\hline
\end{tabular}

hypothesis, we simulated 10000 PN spectra starting from a twotemperature model that reflects the average observed properties of the studied sample, i.e. $N_{\mathrm{H}}, T_{1}, T_{2}, E M_{2} / E M_{1}$ and elemental abundances equal to the medians of the relevant observed distributions (Table 3). The total number of counts in the 10000 simulated spectra reflects the distribution of counts observed in the real spectra of the 20 TMC members. Subsequently, we fitted these spectra with a 2-T model with the same (13) free parameters as before.

The result of this test is reported in Fig. 4, which shows the distributions of the abundances (in the upper panel) and the abundances normalized to iron (in the lower panel) obtained from the real data, compared with the central $90 \%$ ranges of values obtained from the simulations, for each element. In both panels, the spread of the values derived from the real data is comparable to that indicated by the simulations, for all elements. One exception is the $\mathrm{Ne} / \mathrm{Fe}$ abundance ratio, whose distribution is significantly larger in the case of the real data. This is better quantified by a K-S test, which shows that for the case of the $\mathrm{Ne} / \mathrm{Fe}$ ratio the two distributions are different with a significance of more than $3 \sigma$. The range of $\mathrm{O} / \mathrm{Fe}$ ratios also appears to exceed the $90 \%$ bounds of the range of the simulations, however, the difference between the two distributions is not statistically significant (K-S probability of undistinguishable distributions equal to $7 \%$ ). The result concerning the $\mathrm{Ne} / \mathrm{Fe}$ ratio is intriguing and deserves to be further investigated because neon and iron are chemical elements that have different properties related to their tendency to form dust grains, with the volatile noble gas neon remaining in the gaseous phase and iron more easily condensing in grains. Moreover, Ne is a high-FIP element, while Fe is a low-FIP element. In Sect. 4.2 we discuss the Ne/Fe ratio for the stars of the present sample together with some possibilities that could explain the above result.

In Fig. 5 we separate the abundance distributions of the classical and the weak-lined T Tauri stars: the plot shows that there is no significant difference between the distributions for the two classes of sources, indicating that both the thermal properties (Fig. 2) and chemical composition of the X-ray-emitting plasma are similar.

\section{Discussion}

\subsection{Medians of the abundance distributions}

A robust result of this analysis is the low iron abundances of the $\mathrm{X}$-ray bright Taurus members with respect to the solar photospheric value $(\mathrm{Fe} \simeq 0.2$ solar), since the abundance of this element is generally well-determined and with relatively small uncertainties (as indicated by the simulations). This analysis thus confirms the results of other previous studies on high-luminosity stars (PMS stars and RS CVn binary systems), which indicate a low coronal metallicity, with respect to the solar case, in such active stars (e.g. Maggio et al. 2007; Drake et al. 2001; Huenemoerder et al. 2001; Audard et al. 2003).

In Fig. 3 we also report the fixed values of abundances used by Güdel et al. (2007) for fitting the EPIC spectra of more than a hundred Taurus members, with the aim of deriving absorption
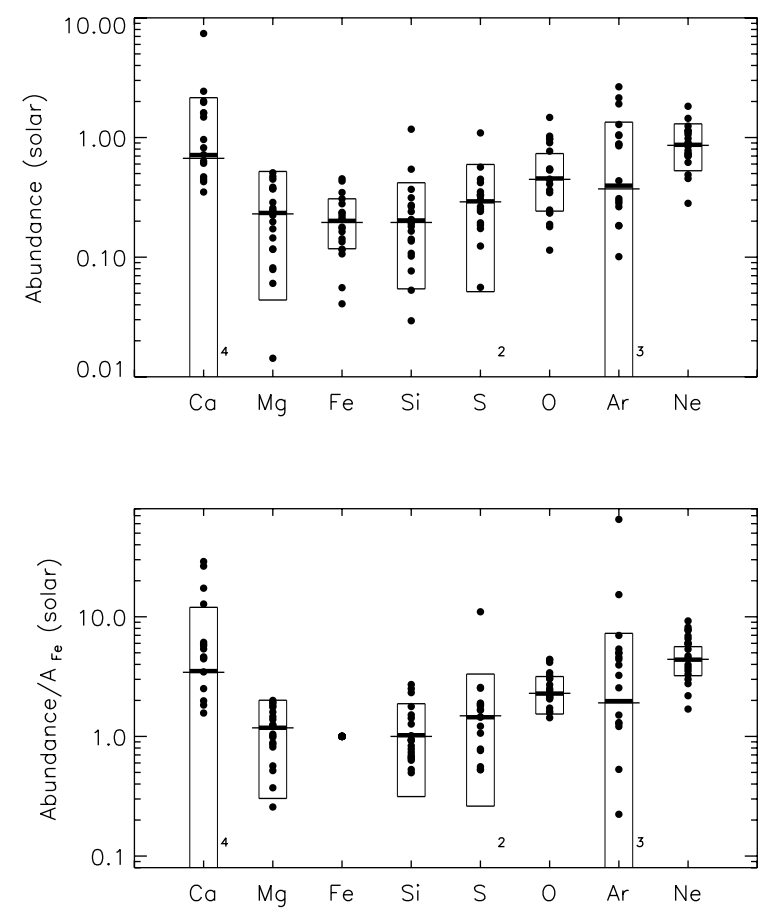

Fig. 4. Distributions of abundances (upper panel) and abundances relative to iron (lower panel) found for the 20 TMC members, compared with the results of simulations (see text). The boxes contain the central $90 \%$ of the abundance values obtained from the simulations, the median being indicated by the thick black segment within each box. The thin, longer segments mark the medians of the abundance distributions derived from real data; they are practically coincident with the median values of the simulations. Numbers at the bottom of the plot are as in Fig. 3.

column densities, characteristic plasma temperatures and X-ray luminosities. The values adopted by these authors (see also Appendix A) were chosen as representative of the coronal abundances in active stars. Our detailed analysis confirms the validity of this assumption, as the medians of the abundance distributions for all elements are very similar to the previously adopted values, with the possible exception of calcium.

In Sect. 3.2 we showed that the medians of the abundance distributions follow a pattern vs. FIP similar to that derived from the analysis of high-resolution X-ray spectra for a number of active stars (Sanz-Forcada et al. 2003; Argiroffi et al. 2004; Scelsi et al. 2004; Argiroffi et al. 2005; Scelsi et al. 2005; Telleschi et al. 2005). Given the large uncertainties of the Ca abundances, our analysis might also suggest a systematic pattern of increasing abundances for increasing FIP. However, such a pattern is obtained by adopting solar photospheric abundances as reference, while any FIP-related effect should be studied by comparison with the stellar photospheric abundances, which unfortunately are not available for any of the stars in our sample. Measuring abundances in stellar photospheres is a difficult task due to the limited quality of stellar optical spectra, uncertainties in stellar parameters (gravity, effective temperature, macroturbulence) and atmospheric models (LTE approximations), line 


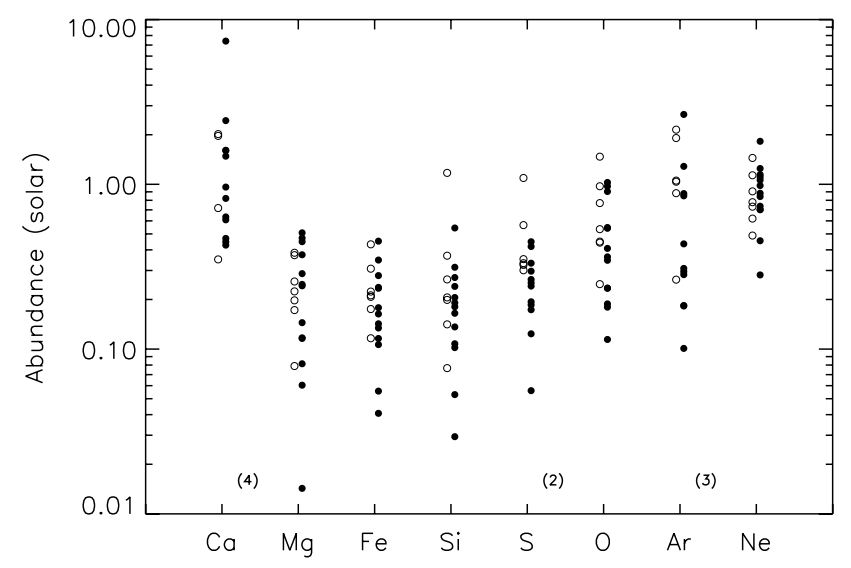

Fig. 5. Scatter plot of abundances obtained for the classical (open circles) and weak-lined (filled circles) $\mathrm{T}$ Tauri stars in the studied sample. Numbers at the bottom of the plot are as in Fig. 3.

blending (especially for cool M-type stars), and rotational broadening effects, which is typically the case of active young stars. Furthermore, the effects of possible stellar spots and of the chromospheric activity on abundance determinations are not well understood, and binarity requires the non-trivial procedure of untangling composite spectra. An assumption usually adopted is that stars in the solar neighbourhood have photospheric abundances similar to the solar ones because they belong to the same galactic population. Moreover, we expect that stars younger than the Sun have on average higher metallicities because they have formed from material enriched in high- $Z$ elements by the remnants of an older stellar population. However, extensive surveys show that there is a large scatter in metallicity among stars of similar age (Nordström et al. 2004). Sanz-Forcada et al. (2004) studied the coronal abundances of four stars with accurately known photospheric abundances and showed that the pattern of coronal-to-photospheric abundance ratios vs. FIP can be different if stellar or solar photospheric abundances are used. The recent study by Maggio et al. (2007) on the X-ray brightest stars in Orion also indicates that a clear trend vs. FIP is no longer observed when the photospheric abundances of the Orion stars or the composition of the gaseous phase in the nebula are considered instead of the solar ones. On the other hand, SanzForcada et al. (2003) derived coronal abundances that are not photospheric for AB Dor, which has well measured photospheric abundances, and Telleschi et al. (2005) found the FIP and inverse FIP patterns in six solar analogs for which several photospheric abundances are known. It is important to dedicate more observing time to collecting optical spectra in order to measure stellar photosperic abundances and hence to address in a proper manner the issue of any FIP-related difference between the photospheric and coronal composition.

\subsection{Ne/Fe abundance ratio}

We now return to the spread of abundances found for the sample stars, for each element. The only quantity showing a spread of the values larger than expected from the simulations is the $\mathrm{Ne} / \mathrm{Fe}$ ratio (Fig. 4), which varies between $\sim 2$ and $\sim 9$, with a median around $\mathrm{Ne} / \mathrm{Fe} \sim 5$. The ratio between the abundance of a given element and that of iron is generally better constrained than absolute abundance values since systematic errors in the elemental and iron abundances due, for example, to a wrong estimate of the continum emission, usually go in the same direction and can compensate when calculating their ratio. The result we obtained deserves to be investigated for several reasons.

A plausible assumption is that the photospheric abundances of iron and neon are about the same in all the stars studied here because they belong to the same region and hence they were born from the same cloud material; the $\mathrm{Ne} / \mathrm{Fe}$ ratio in their coronal plasma could then vary depending on the stellar activity level if FIP-related effects are really present in stellar coronae, as suggested by previous studies (Brinkman et al. 2001; Audard et al. 2003). The X-ray luminosities of the sample stars are in the range $10^{30}-10^{31} \mathrm{erg} \mathrm{s}^{-1}$, therefore the study is biased towards active TMC members. However, Audard et al. (2003) found that a transition from an inverse FIP effect to a flat pattern with decreasing coronal activity is visible in a sample of RS CVn binary systems spanning about the same $\mathrm{X}$-ray luminosity range as that of the present study. In particular, they report an anticorrelation between $\mathrm{Fe} / \mathrm{O}$ vs. the average coronal temperature $T_{\mathrm{av}}$ of five active RS CVn-like systems, as well as a constant value of the $\mathrm{Ne} / \mathrm{O}$ ratio, which translates into a correlation between $\mathrm{Ne} / \mathrm{Fe}$ and $T_{\mathrm{av}}$. When adding a sample of solar analogs studied by Güdel et al. (2002), reaching lower activity levels, such a correlation becomes much more evident and a trend from an inverse to a normal FIP effect is seen.

We plot in Fig. 6 the Ne/Fe ratio for our sample of TMC stars as a function of various activity indicators (X-ray luminosity, $L_{\mathrm{X}}$, average temperature, $T_{\mathrm{av}}$, and surface X-ray flux, $F_{\mathrm{X}}$ ), in order to search for possible transitions from a FIP effect to an inverse FIP effect with increasing activity. However, no significant correlation of $\mathrm{Ne} / \mathrm{Fe}$ with any of the considered parameters is found from the available data, which therefore does not support the existence of different FIP-related effects in the coronae of the studied stars.

However, we also note an apparent drop of the $\mathrm{Ne} / \mathrm{Fe}$ ratios for stars with average temperature $T_{\mathrm{av}} \gtrsim 15 \mathrm{MK}$. Based on the analysis of RGS spectra of a few stars in the XEST project, Telleschi et al. (2007) also found a bimodality in the Fe abundance and $\mathrm{Ne} / \mathrm{Fe}$ ratio for their sample stars, although this behaviour was observed to be linked to the spectral type. In particular, the above authors found larger Fe abundances and lower $\mathrm{Ne} / \mathrm{Fe}$ ratios in three G-type stars with respect to six K- and M-type stars. To check their result, we divided our sample into two groups, i.e. stars with spectral type earlier and later than K2 (7 sources belong to the first group, while 12 stars have spectral type later than K2, the spectral type of the star RXJ 0422.1+1934 not being available), and we observe in Fig. 6 that most of the stars cooler than $15 \mathrm{MK}$ have indeed spectral type later than K2. The K-S test gives $0.15 \%$ probability that the distributions of $T_{\mathrm{av}}$ for the two groups are drawn from the same parent distribution. We note that this difference derives from earlier-type stars having typically higher $T_{2}$ and $E M_{2} / E M_{1}$ values.

Figure 7 shows the cumulative distributions of the iron and neon abundances and of the $\mathrm{Ne} / \mathrm{Fe}$ ratio for the stars of the two groups: there is a systematic difference in the neon abundance against the spectral type, and an even more clear evidence for lower Fe abundances in $\mathrm{K}$ - and M-type stars, which implies larger $\mathrm{Ne} / \mathrm{Fe}$ ratios than in earlier-type stars. The probability that the $\mathrm{Fe}$ distributions for the two groups are not distinguishable is $0.6 \%$; for the cases of the $\mathrm{Ne} / \mathrm{Fe}$ and $\mathrm{Ne}$ distributions, the probabilities are $8 \%$ and $17 \%$, respectively. Franciosini et al. (2007) studied the G-type Taurus member SU Aur ( $\left.T_{\mathrm{av}} \sim 20 \mathrm{MK}\right)$ and found $\mathrm{Fe} \sim 0.6$ times the solar value and $\mathrm{Ne}$ abundance about equal to the solar one, with independent analyses of MOS and RGS data. If we also consider the abundances obtained for 

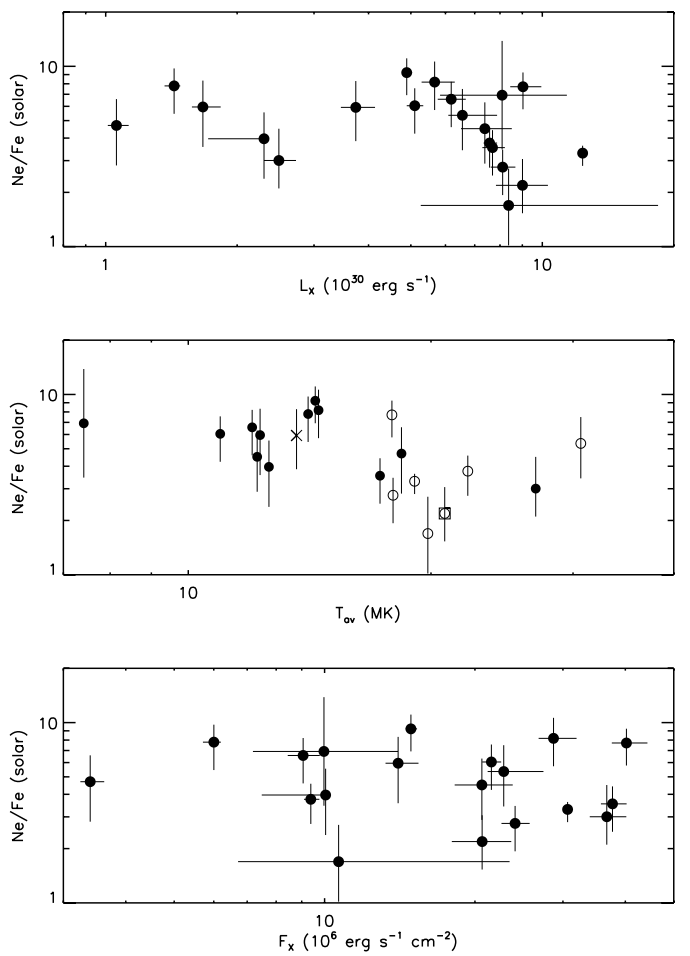

Fig. 6. $\mathrm{Ne} / \mathrm{Fe}$ ratios of all sample stars plotted as a function of three activity indicators: X-ray luminosity (upper panel), average temperature (middle panel) and surface X-ray flux (lower panel). In the plot of $\mathrm{Ne} / \mathrm{Fe}$ vs. $T_{\mathrm{av}}$, different spectral types are shown (see text): stars with spectral type later than $\mathrm{K} 2$ are plotted as filled circles, while open circles refer to spectral type earlier than K2 (an open square superimposed to the circle marks the Herbig Be star V892 Tau); the cross represents the WTTS RXJ0422.1+1934, whose spectral type is not available.

SU Aurigae, the result for $\mathrm{Fe}$ and for $\mathrm{Ne} / \mathrm{Fe}$ is statistically more significant; the three K-S test probabilities mentioned above are $0.4 \%(\mathrm{Fe}), 4.5 \%(\mathrm{Ne} / \mathrm{Fe})$ and $29 \%(\mathrm{Ne})$. In this calculation we have assumed that the spectral type of the companion of V928 Tau (the probable source of X-rays) is K2; we have verified that the above conclusions do not change assuming that this star has a spectral type later than $\mathrm{K} 2$.

A possible dependence of $\mathrm{Fe}$ and $\mathrm{Ne} / \mathrm{Fe}$ on the spectral type has not been claimed in the past, before the report by Telleschi et al. (2007), and high-quality spectra of a larger sample of stars are needed to confirm this finding. Since the stars in our sample with spectral type later than $\mathrm{K} 2$ have, on average, cooler coronae, this result may also reflect a dependence on $T_{\text {av }}$ (although this is not evident in the Telleschi et al. sample). However, for the stars with the highest $T_{\text {av }}$ (in the range 10-30MK), such a dependence would be in the opposite direction with respect to that found by Audard et al. (2003), who report increasing Ne/Fe with increasing $T_{\text {av }}$ in the range $\sim 4-15 \mathrm{MK}$.

In the light of the above result, we run simulations analogous to those described in Sect. 3.2, but taking into account a possible bimodality of the iron abundance with the spectral type, i.e. we assumed $\mathrm{Fe}=0.24$ and $\mathrm{Fe}=0.15$ for stars with spectral type earlier and later than K2, respectively. Figure 8, analogous to the lower panel of Fig. 4, shows that the spread of $\mathrm{Ne} / \mathrm{Fe}$ in real data can be explained in this way (K-S test probability $=32 \%$ ).

Another issue of potential importance is that $\mathrm{Fe}$ forms grains much more readily than Ne: as explained in Sect. 1, if iron settles in dust grains in the disk of CTTSs and the dust and gas components are separated for some reason, X-ray emission from accreting stars may reveal a metal-depleted plasma. We note that such a result may be expected if accretion-driven emission dominates over a coronal component, since in this case the abundances we are measuring are essentially those of the accreted plasma.

The X-ray emitting plasmas of the stars in our sample have rather high average temperature $(\gtrsim 10 \mathrm{MK})$, and similar properties in both the CTTSs and the WTTSs, indicating that we are probably seeing a dominant contribution from a coronal emission. Figure 9 shows the $\mathrm{Ne} / \mathrm{Fe}$ ratio of our sample stars as a function of two stellar parameters linked to the presence of circumstellar material, i.e. $N_{\mathrm{H}}$ and the accretion rate. For comparison, we also report the ratios for the two classical T Tauri stars TW Hydrae (Robrade \& Schmitt 2006) and MP Muscae (Argiroffi et al. 2007); for the CTTS BP Tauri, previously studied by other authors, we note that our $\mathrm{Ne} / \mathrm{Fe}$ ratio is in agreement with that found by Robrade \& Schmitt (2006). As expected, we do not find evidence of any relation between $\mathrm{Ne} / \mathrm{Fe}$ and these two parameters; moreover, as shown in Fig. 9, high Ne/Fe ratios may be present both in CTTSs and in WTTSs and are therefore not a peculiar characteristic of accreting stars. This is consistent with the findings of Telleschi et al. (2007) that high $\mathrm{Ne} / \mathrm{Fe}$ ratios are not present in all accreting stars.

\section{3. $\mathrm{Ne} / \mathrm{O}$ ratio}

The Ne/O ratio shares some similarities with the $\mathrm{Ne} / \mathrm{Fe}$ one, in that oxygen can easily combine with dust grains; Drake \& Testa (2005) analyzed high-resolution Chandra spectra from a variety of stars (dwarfs, giants, multiple systems, subgiants) and found that the $\mathrm{Ne} / \mathrm{O}$ ratio is remarkably constant in stellar coronae (Ne/O 0.4, the solar value by Anders \& Grevesse 1989 being $\sim 0.14$ ). This is also true for the classical T Tauri stars BP Tauri and TWA 5, but not for the CTTS TW Hydrae, whose higher $\mathrm{Ne} / \mathrm{O}$ value $(\sim 1)$ was interpreted by Drake et al. (2005) in terms of depletion of grain-forming elements in the X-ray emitting gas that is accreting onto the star: such a depletion is supposed to be more pronounced in the case of the more evolved and older circumstellar disk of TW Hydrae, where coagulation of grains into larger bodies orbiting the star could have happened; in the younger CTTS BP Tauri, where part of the emission is accretiondriven, the dust particles could still be small and can release, after heating, the elements locked into the grains. However, very recently Argiroffi et al. (2007) obtained Ne/O $~ 0.5$ for the CTTS MP Muscae, which is even older than TW Hydrae, but still shows evidence for a substantial contribution to the X-ray emission from accretion shocks in the high plasma density derived from the analysis of the $\mathrm{O}$ VII triplet; the average plasma temperature of this star is relatively high and hence the derived abundances are likely affected by the coronal emission. What is also emerging from these abundance studies is the need for a criterion to understand which abundances (coronal or accretionrelated) we are measuring in these stars, perhaps also with the help of modeling. For the stars we studied here, the $\mathrm{Ne} / \mathrm{O}$ ratio is affected by large uncertainties in those sources with high absorption, because of the difficulty of constraining the $\mathrm{O}$ abundance in this case. If we consider the 12 stars with $N_{\mathrm{H}} \leq 3 \times 10^{21} \mathrm{~cm}^{-2}$, we find that the $\mathrm{Ne} / \mathrm{O}$ ratio is in the range $\sim 0.2-0.6$, with the values equally spread around $\sim 0.4$ (Fig. 10). In particular, this is true also for the classical T Tauri stars DN Tauri, DH Tauri, T Tauri, and BP Tauri ${ }^{2}$, among the $12 \mathrm{we}$ are considering. They are rather

\footnotetext{
2 The value $\mathrm{Ne} / \mathrm{O}$ found in this study for BP Tauri is in good agreement with that obtained by Robrade \& Schmitt (2006).
} 

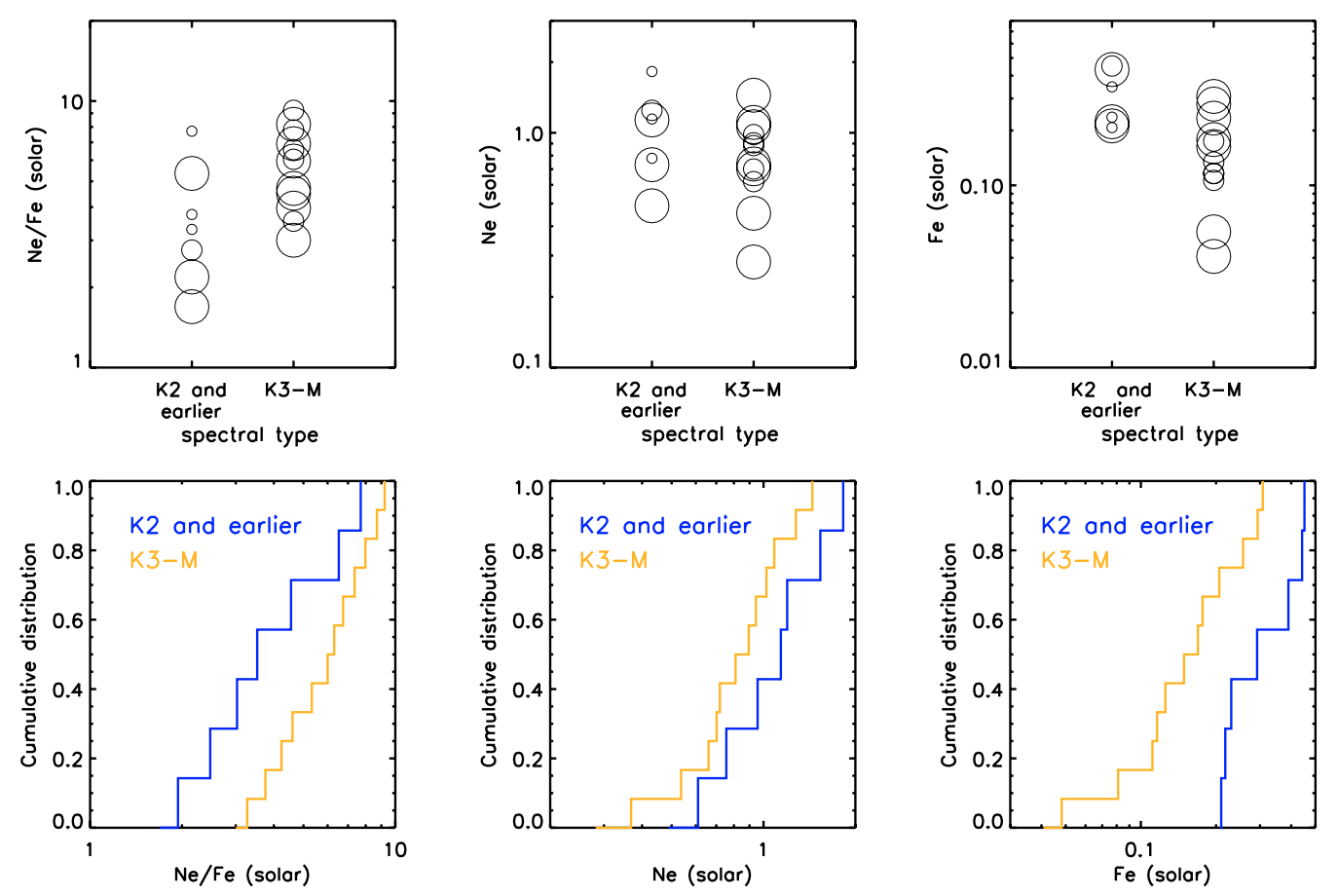

Fig. 7. Ne and $\mathrm{Fe}$ abundance distributions and $\mathrm{Ne} / \mathrm{Fe}$ ratios for stars with spectral type earlier and later than $\mathrm{K} 2$ (three upper panels); the circle size decreases with increasing number of total counts in the PN spectrum. In the three lower panels the relevant cumulative distributions are shown.

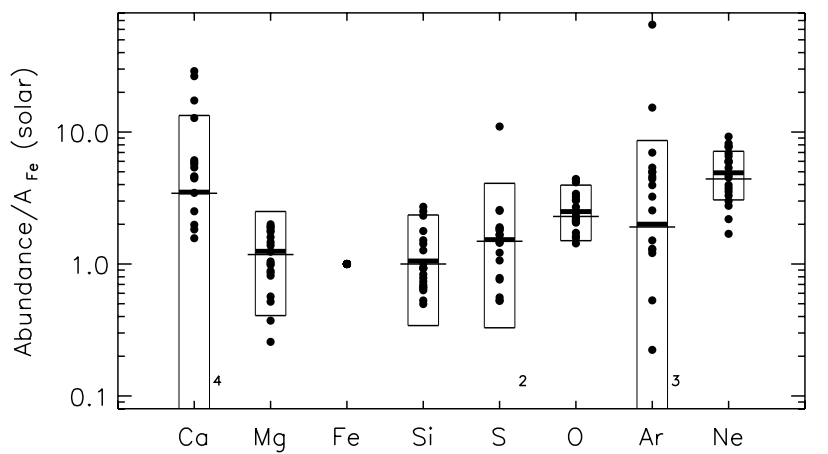

Fig. 8. Distributions of abundances relative to iron for the $20 \mathrm{TMC}$ members compared with the results of simulations taking into account a possible bimodality of the iron abundance (see text). Symbols and lines are as in Fig. 4.

young, with ages in the range $\sim 1-2.7 \mathrm{Myr}$, and their high average plasma temperatures imply a large coronal contribution, as already discussed. Therefore, our result does not go against the scenario mentioned above.

\section{Summary and conclusions}

The thermal properties and chemical composition of the X-ray emitting plasma in a sample of bright Taurus members have been studied in this work with the aim of investigating possible systematic differences between classical and weak-lined $\mathrm{T}$ Tauri stars, and of understanding whether the pattern of abundances in such stars is related to some stellar or environmental characteristics. We used data from the XEST survey; since there are very few useful RGS spectra of Taurus members, we used the PN data in order to enlarge the sample to be analyzed as much as possible. Preliminary extensive simulations showed that PN spectra with $\sim 5000$ counts or more in the $0.3-10 \mathrm{keV}$ band can provide reliable estimates of elemental abundances and $68 \%$ statistical errors usually within a factor $\sim 2$ for most of the elements; exceptions may be $\mathrm{O}, \mathrm{Ca}$ and $\mathrm{Ar}$ in some cases, depending on the statistics and source absorption. The sample of stars considered for this work therefore consists of 20 TMC members: 13 weaklined T Tauri stars and 7 accreting stars, having at least 4500 total counts in their spectra. Their medium-resolution spectra were globally fitted with an absorbed two-temperature thermal model with variable abundances of $\mathrm{O}, \mathrm{Ne}, \mathrm{Mg}, \mathrm{Si}, \mathrm{S}, \mathrm{Ar}, \mathrm{Ca}$ and $\mathrm{Fe}$.

The temperatures of the two components were found in the ranges $\sim 5-10 \mathrm{MK}$ and $\sim 20-40 \mathrm{MK}$, and the hot-to-cool emission measure ratios range between $\sim 0.8$ and 4 . Average temperatures are in the range $\sim 8-30 \mathrm{MK}$, with the classical T Tauri stars being slightly hotter than the WTTSs.

The spread of the 20 abundance values for each element (Fig. 3) is more or less compatible with the errors obtained from the simulations; a posteriori tests, also based on simulations, have shown that our results are consistent with these TMC stars sharing a unique set of abundances, with the possible exception of iron.

The main conclusions from the present work are summarized as follows. Hot coronal plasma is responsible for most of the X-ray emission from both the CTTSs and WTTSs studied here. The coronal plasma has similar properties in the two classes of T Tauri stars, in particular similar characteristic temperatures and chemical composition. Late-type stars therefore develop hot coronae early in their life (within $\sim 1 \mathrm{Myr}$ ), whose properties change little during the following pre-main sequence evolution, at least up to an age of $\sim 10 \mathrm{Myr}$. The reason why the coronal emission appears suppressed in some CTTSs, such as TW Hydrae, and not in most of the others is to be clarified with future work.

The available data do not allow us to evaluate quantitatively the presence, in the CTTSs considered here, of a cool X-ray emission excess that might be present due to shock-heating. The iron abundance is significantly subsolar $(\sim 0.1-0.5$ times the 

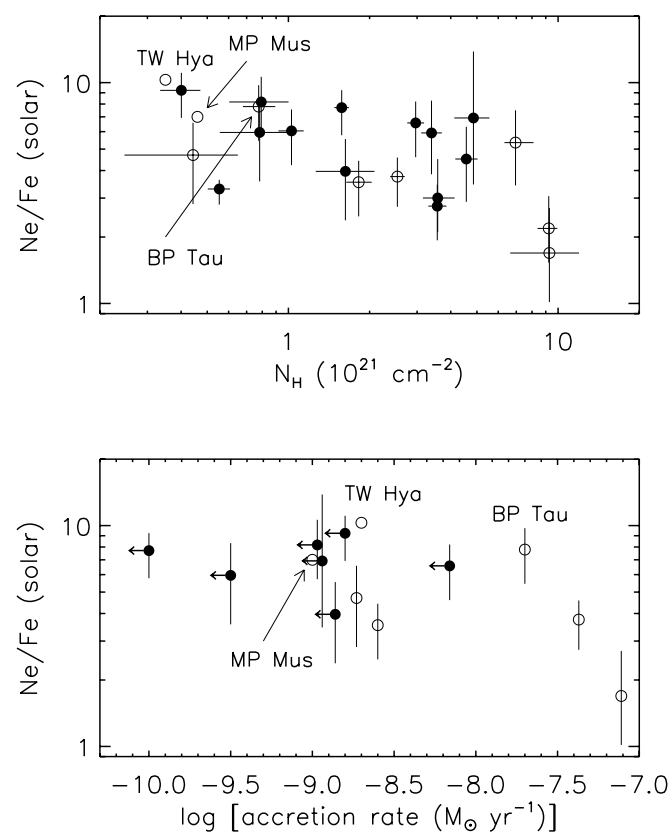

Fig. 9. $\mathrm{Ne} / \mathrm{Fe}$ ratios as a function of the photoelectric absorption (upper panel) and accretion rate (lower panel). Errors are $1 \sigma$. Filled and open circles mark WTTSs and CTTSs, respectively. In both plots, the positions of the two CTTSs TW Hydrae and MP Muscae are shown as open circles without error bars. In the lower panel, only stars with measured accretion rates are shown; all WTTSs have upper limits indicated by left arrows. Accretion rates for the TMC members, for TW Hydrae and for MP Muscae are taken from the tables in Güdel et al. (2007), from Alencar \& Batalha (2002) and from Sacco G. G. (private comunication), respectively.

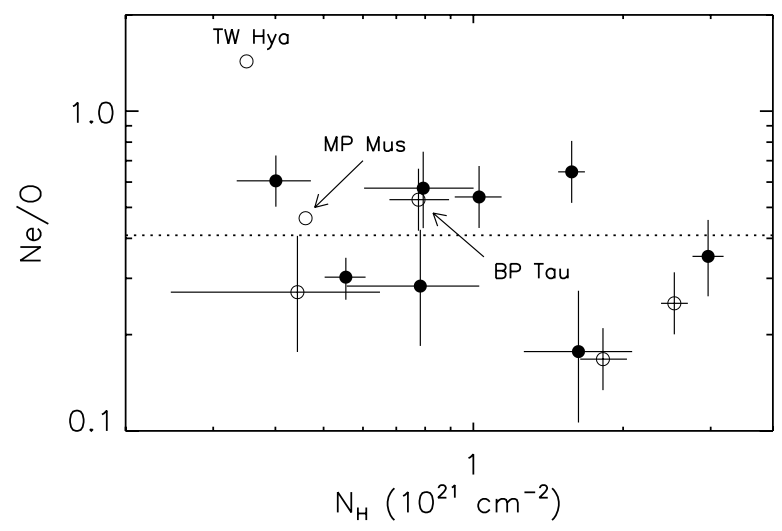

Fig. 10. Ne/O ratios as a function of the photoelectric absorption, for stars with $N_{\mathrm{H}} \leq 3 \times 10^{21} \mathrm{~cm}^{-2}$. Errors are $1 \sigma$. Filled and open circles mark WTTSs and CTTSs, respectively. For comparison, the positions of the two CTTSs TW Hydrae and MP Muscae are also shown (open circles without error bars). The dotted line marks the average value of 0.41 found by Drake \& Testa (2005) for stars of various activity levels.

solar photospheric value) in all the sample stars. Since the abundance of this element is the best-constrained one, this result is rather robust and confirms previous finding for PMS and active stars.

We found indication of coronae in G-type and early K-type stars that are hotter than in stars with later spectral type. We also find strong support for a bi-modality of the Fe abundance (and the $\mathrm{Ne} / \mathrm{Fe}$ ratio), depending on the spectral type (or perhaps on the average temperature), with higher $\mathrm{Fe}$ values and lower $\mathrm{Ne} / \mathrm{Fe}$ ratios for G-type and early K-type stars compared to stars of later spectral type. The latter result supports the findings from high-resolution spectroscopy by Telleschi et al. (2007). A larger sample is needed, however, to find out if this trend is merely the result of the limited statistics presently available.

The analysis of the abundance distributions of this sample of bright TMC members did not reveal any significant difference in the abundances related to the presence of accretion/circumstellar material. $\mathrm{Ne} / \mathrm{Fe}$ abundance ratios between 2 and 9 times the solar value are found both for the classical and the weak-lined T Tauri stars, confirming the findings by Telleschi et al. (2007) based on a smaller sample of high-resolution X-ray spectra of T Tauri stars. Regarding the Ne/O ratio, we do not observe in the classical T Tauri stars a behaviour analogous to that of TW Hydrae, all values being below $\sim 0.6$.

Acknowledgements. We acknowledge financial support by the International Space Science Institute (ISSI) in Bern to the XMM-Newton XEST team. The Palermo group acknowledges financial contribution from contract ASI-INAF I/023/05/0. X-ray astronomy research at PSI has been supported by the Swiss National Science Foundation (grants 20-66875.01 and 20-109255/1). This research is based on observations obtained with XMM-Newton, an ESA science mission with instruments and contributions directly funded by ESA member states and the USA (NASA).

\section{Appendix A: Simulations}

In this section we describe the simulations we run before fitting the real data in order to assess the performances of the PN regarding abundance determinations. This part of the investigation can be summarized as follows:

1. Fourteen input coronal models were chosen, each consisting of an EM distribution and a set of elemental abundances, plus interstellar absorption.

2. Three different numbers of source counts were considered: $\sim 5000,10000$ and 20000 counts in the $0.3-10 \mathrm{keV}$ band of the PN spectrum. This choice is motivated by the fact that most of the brightest TMC members have total PN counts within the above range; moreover, previous fittings of Taurus members with less than 5000 counts in the PN, using 2-T models with a fixed set of abundances (Güdel et al. 2007) already provided good descriptions of the PN spectra, a result which implies no need to treat the abundances as free parameters.

3. For each simulated observation, i.e. for each coronal model at each number of total counts, 1000 PN spectra were simulated (using the standard PN response function and assuming no background contamination), which have a Poissonian distribution of the total number of counts.

4. For each simulated observation, the $1000 \mathrm{PN}$ spectra were fitted with an automatic routine, using a 2-T coronal model with the following starting values for the fitting parameters: abundances and $N_{\mathrm{H}}$ equal to the values of the input coronal model, $T_{1}$ and $T_{2}$ given by the results of interactive fittings of a few simulated spectra for each of the fourteen input models.

Moreover, for the input models 1 and 2 (see Table A.1) the fitting of simulated spectra was repeated for each of the three values of the total counts, starting from different initial values of $N_{\mathrm{H}}$ and temperatures, and initial abundances equal to the solar values: for each of these 6 simulations, 300 spectra were automatically 
fitted by performing an extensive exploration of the $N_{\mathrm{H}}, T_{1}$ and $T_{2}$ space so as to simulate the approach to real data. The results obtained in this way are practically undistinguishable from those derived with the procedure described in item \#4 above. For the input models 9 and 10, non-solar abundances as starting values for the fitting of simulated spectra were also used. Again, the results in these cases are very similar to those obtained with the procedure in item \#4.

Note that using the same instrumental response both to simulate and to fit the spectra implies that this investigation leads to estimate the errors in the determination of the plasma parameters due to the photon counting statistics and to the instrument spectral resolution. The method cannot provide estimates of possible systematic errors due to uncertainties in the instrumental calibration and in the atomic parameters used to calculate theoretical plasma emissivities.

The input coronal models were chosen so as to explore characteristic absorption values and physical conditions of the coronal plasma, in terms of emission measure distribution (EMD) and abundances, among those commonly found in the literature for active T Tauri stars. For the abundances, some extreme cases were also explored (see below). The emission measure distributions we have considered for these simulations are defined in the temperature range $10^{6} \mathrm{~K} \leq T \leq 10^{7.5} \mathrm{~K}$ as:

$$
E M D \propto \begin{cases}T^{\alpha} & 10^{6} \mathrm{~K} \leq T \leq T_{\text {peak }} \\ T^{-\beta} & T_{\text {peak }} \mathrm{K} \leq T \leq 10^{7.5} \mathrm{~K} .\end{cases}
$$

Based on EMD reconstructions with high-resolution spectra of intermediate- and high-activity stars by Laming \& Drake (1999); Argiroffi et al. (2004); Scelsi et al. (2005); Argiroffi et al. (2005); Telleschi et al. (2005); Güdel et al. (2007), we chose the values of $\alpha$ and $\beta$ in the range 2-5 and $1-2$, respectively, while two values of $T_{\text {peak }}$ were explored $\left(10^{6.7} \mathrm{~K}\right.$ and $\left.10^{7} \mathrm{~K}\right)$. Four sets of abundances were adopted: (i) the abundance set used by Güdel et al. (2007) to fit the spectra of known TMC members (hereafter TMC set): with respect to the solar photospheric values by Anders \& Grevesse (1989), the abundances of this set are: $\mathrm{C}=0.45, \mathrm{~N}=0.788$, $\mathrm{O}=0.426, \quad \mathrm{Ne}=0.832, \quad \mathrm{Mg}=0.263, \quad \mathrm{Al}=0.5, \quad \mathrm{Si}=0.309$, $\mathrm{S}=0.417, \mathrm{Ar}=0.55, \mathrm{Ca}=0.195, \mathrm{Fe}=0.195, \mathrm{Ni}=0.195$; (ii) all abundances equal to 0.2 times the solar photospheric values of Anders \& Grevesse (1989); (iii) solar photospheric values for all abundances, except for iron ( $=0.2$ solar); (iv) the TMC set, except for iron $(=0.5$ solar $)$. We finally considered two absorption levels: "low" absorption with $N_{\mathrm{H}}=10^{21} \mathrm{~cm}^{-2}$, and "high" absorption with $N_{\mathrm{H}}=10^{22} \mathrm{~cm}^{-2}$. The fourteen input models are listed in Table A.1.

The two-temperature thermal models used to fit the simulated spectra are based on the APEC emissivity code and have the following 13 free parameters: the interstellar hydrogen column density $\left(N_{\mathrm{H}}\right)$, the temperature and emission measure of each plasma component, and the abundances of $\mathrm{O}, \mathrm{Ne}, \mathrm{Fe}, \mathrm{Mg}, \mathrm{Si}, \mathrm{S}$, $\mathrm{Ar}, \mathrm{Ca}$. We fixed the abundances of $\mathrm{C}, \mathrm{N}$ and $\mathrm{Ni}$ to the respective values in the TMC set, because they are generally not wellconstrained from $\mathrm{PN}$ fitting; in fact, the $\mathrm{C}$ and $\mathrm{N}$ lines fall at low energies where the PN resolution is quite poor (and moreover the PN calibration is probably uncertain), while the Ni lines are blended with the (usually) stronger iron and neon lines.

As representative examples of the results of all simulations, we report in Fig. A.1 those obtained for the abundances in terms of medians of the distributions of the 1000 best-fit values, and error bars corresponding to the central $90 \%$ of the distributions, for ten different cases illustrating the effects of different input
Table A.1. Input models for simulations. The TMC set is the abundance set used by Güdel et al. (2007) (see text). "Low" and "high" absorption mean $N_{\mathrm{H}}=10^{21} \mathrm{~cm}^{-2}$ and $N_{\mathrm{H}}=10^{22} \mathrm{~cm}^{-2}$, respectively.

\begin{tabular}{lccccc}
\hline \hline Model & $\log T_{\text {peak }}$ & $\alpha$ & $\beta$ & Abundances & Absorption \\
\hline 1 & 7 & 3 & 2 & TMC & low \\
2 & 7 & 3 & 2 & TMC & high \\
3 & 7 & 5 & 2 & TMC & low \\
4 & 7 & 5 & 2 & TMC & high \\
5 & 7 & 2 & 1 & TMC & low \\
6 & 7 & 2 & 1 & TMC & high \\
7 & 7 & 3 & 2 & 0.2 solar & low \\
8 & 7 & 3 & 2 & 0.2 solar & high \\
9 & 7 & 3 & 2 & solar (Fe $=0.2$ solar $)$ & low \\
10 & 7 & 3 & 2 & solar (Fe $=0.2$ solar $)$ & high \\
11 & 7 & 3 & 2 & TMC (Fe $=0.5$ solar) & low \\
12 & 7 & 3 & 2 & TMC (Fe $=0.5$ solar) & high \\
13 & 6.7 & 2 & 1 & TMC & low \\
14 & 6.7 & 2 & 1 & TMC & high \\
\hline
\end{tabular}

models, statistics, absorptions and choice of starting abundance values for the fittings.

The results indicated that, for all the cases analyzed here, the derived abundances are generally in good agreement with the values of the input models. The medians of the abundance distributions obtained from fitting simulated spectra are, for all elements, compatible with the input value within $90 \%$ statistical errors, and within the $68 \%$ errors in the great majority of the cases. Some systematic effects must be noted in a few cases: the Ne abundance is overestimated by a factor $\sim 2$ in models 7 and 11, while Fe and $\mathrm{O}$ are underestimated by about the same factor in model 13.

As to the magnitude of the statistical uncertainties, errors at $90 \%$ confidence level within a factor $\sim 2.5$ are obtained for most of the elements using PN spectra with 5000 counts, and within a factor $\sim 2$ with 10000 counts. Exceptions are oxygen, argon and calcium in some cases (see below). Iron is the best-determined element, constrained within a factor $\sim 1.6$ when absorption is relatively low, already with spectra containing 5000 counts. For the other elements, $90 \%$ errors on the medians can be a factor $\sim 1.4$ up to 3 (spectra with 10000 counts), depending on the model.

The abundance of oxygen is very poorly constrained (even for the highest statistics) when the $N_{\mathrm{H}}$ is of the order of $10^{22} \mathrm{~cm}^{-2}$, as one could expect because of the strong attenuation of the spectrum at low energies $(E \lesssim 1 \mathrm{keV}$; recall that the strongest lines of the He-like and H-like ions of oxygen lie at $E \sim 0.6 \mathrm{keV})$. Regarding argon, its abundance can be better constrained in the case of high absorption; this can be easily understood by noting that, for a fixed number of total counts, a larger fraction of all the spectral counts goes in the Ar lines $(\sim 3 \mathrm{keV})$ when the low-energy region of the spectrum is strongly suppressed by the interstellar absorption. Instead, although the strongest $\mathrm{Ca}$ lines fall at $\sim 4.1 \mathrm{keV}$, i.e. close to the Ar lines in the high-energy tail of the spectrum, the abundance of this element is generally ill-constrained, unless the abundance is of the order of the solar value, or higher, and the statistics are high. Since the PN effective area is about the same at $\sim 3 \mathrm{keV}$ and $\sim 4.1 \mathrm{keV}$, the difference with the case of Ar is likely due to the lower emissivity of the calcium lines, compared to those of the Ar lines.

It is important to also note that the results of the simulations are practically the same, in terms of both magnitude of the 

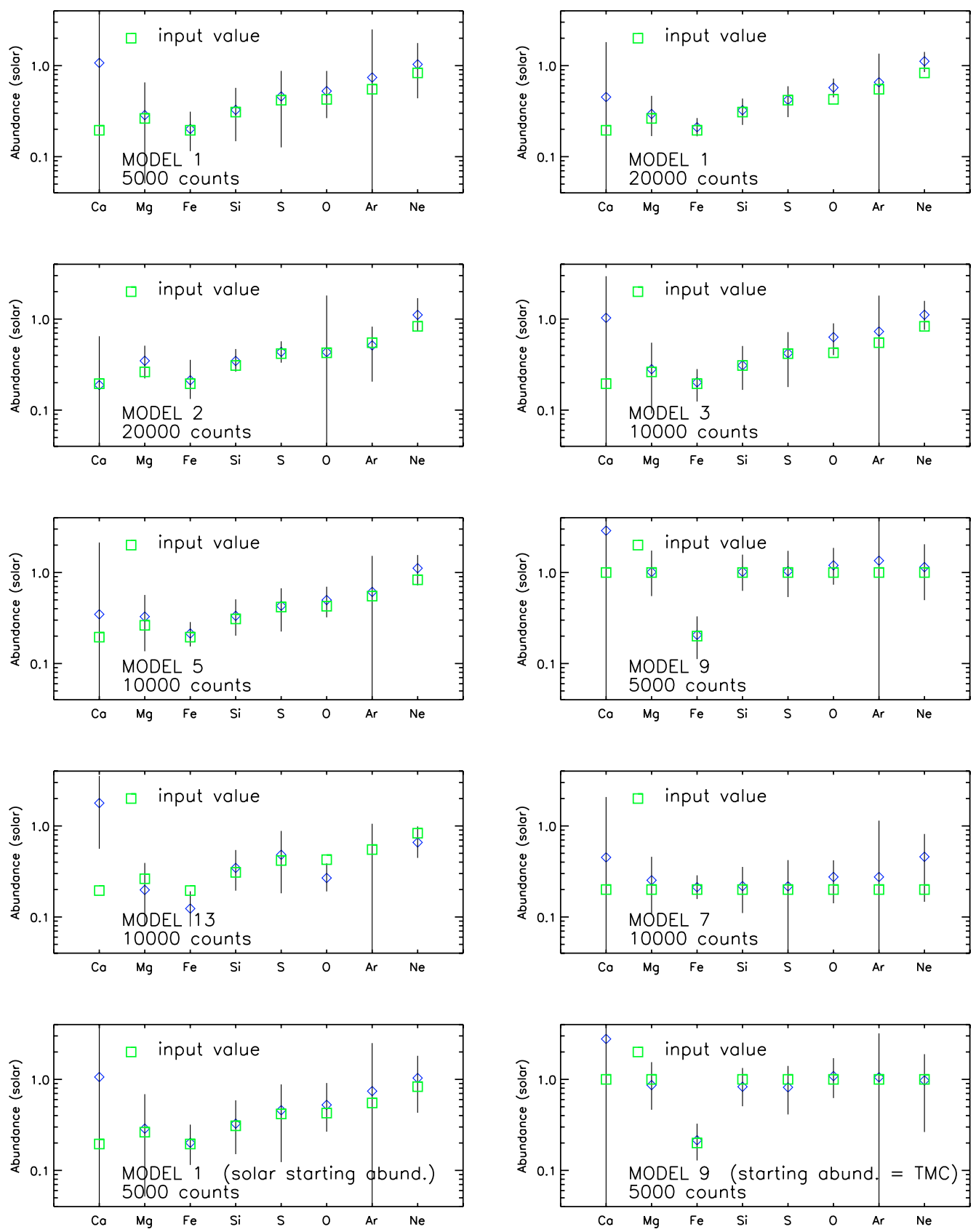

Fig. A.1. Medians (diamonds) and central $90 \%$ of the distributions of the abundances found from eight sample simulations, for different models and/or statistics. The input values of abundances are shown as squares. The input EMDs are equal in models $1,2,7$ and 9 , with $\log T_{\text {peak }}=7$. The EMD of model 3 is steeper, while the EMD of model 5 is flatter. The EMD of model 13 peaks at $\log T_{\text {peak }}=6.7$. The input models have low absorption, with the exception of model 2 . The two bottom panels show the results obtained when the starting abundances for the fitting are different from the "input value" of the relevant model.

errors and agreement between medians of the abundance distributions and input values, regardless of the starting abundance values used to fit the spectra.

Finally, note that the simulations described in this section assumed no background contamination, which may not be negligible at the highest energies. For the bright stars discussed in this paper, we verified that in only a couple of cases the background spectrum dominates over the source spectrum in the energy range $E \gtrsim 5 \mathrm{keV}$, which was therefore excluded from the fitting. To investigate the effect of the exclusion of the Fe XXV $6.7 \mathrm{keV}$ complex on the ability to constrain the iron abundance and the other parameters of the model, we repeated the simulations for the models 1 and 2 by fitting the simulated spectra in the energy range $0.3-5 \mathrm{keV}$. The results obtained in these cases are very similar to those obtained employing a larger spectral range. 


\section{References}

Alencar, S. H. P., \& Batalha, C. 2002, ApJ, 571, 378

Anders, E., \& Grevesse, N. 1989, Geochim. Cosmochim. Acta, 53, 197

Argiroffi, C., Drake, J. J., Maggio, A., et al. 2004, ApJ, 609, 925

Argiroffi, C., Maggio, A., \& Peres, G. 2007, A\&A, 465, L5

Argiroffi, C., Maggio, A., Peres, G., Stelzer, B., \& Neuhäuser, R. 2005, A\&A, 439, 1149

Audard, M., Güdel, M., Sres, A., Raassen, A. J. J., \& Mewe, R. 2003, A\&A, 398, 1137

Brinkman, A. C., Behar, E., Güdel, M., et al. 2001, A\&A, 365, L324

Charnley, S. B. 1997, MNRAS, 291, 455

Drake, J. J. \& Testa, P. 2005, Nature, 436, 525

Drake, J. J., Laming, J. M., \& Widing, K. G. 1997, ApJ, 478, 403

Drake, J. J., Brickhouse, N. S., Kashyap, V., et al. 2001, ApJ, 548, L81

Drake, J. J., Testa, P., \& Hartmann, L. 2005, ApJ, 627, L149

Duchêne, G., Monin, J.-L., Bouvier, J., \& Ménard, F. 1999, A\&A, 351, 954

Favata, F., \& Schmitt, J. H. M. M. 1999, A\&A, 350, 900

Feldman, U. 1992, Physica Scripta Volume T, 46, 202

Franciosini, E., Scelsi, L., Pallavicini, R., \& Audard, M. 2007, A\&A, 471, 951

Güdel, M., Audard, M., \& Sres, A. 2002, in Stellar Coronae in the Chandra and XMM-Newton Era, ed. F.Favata \& J.J.Drake (San Francisco: ASP), ASP Conf. Ser. 277, press

Güdel, M., Briggs, K. R., Arzner, K., et al. 2007, A\&A, 468, 353

Güdel, M., Linsky, J. L., Brown, A., \& Nagase, F. 1999, ApJ, 511, 405

Hartigan, P., \& Kenyon, S. J. 2003, ApJ, 583, 334
Huenemoerder, D. P., Canizares, C. R., \& Schulz, N. S. 2001, ApJ, 559, 1135

König, B., Neuhäuser, R., \& Stelzer, B. 2001, A\&A, 369, 971

Laming, J. M. 2004, ApJ, 614, 1063

Laming, J. M. \& Drake, J. J. 1999, ApJ, 516, 324

Laming, J. M., Drake, J. J., \& Widing, K. G. 1994, BAAS, 26, 866

Laming, J. M., Drake, J. J., \& Widing, K. G. 1996, ApJ, 462, 948

Maggio, A., Flaccomio, E., Favata, F., et al. 2007, ArXiv Astrophysics e-prints Meyer, J.-P. 1985, ApJS, 57, 151

Nordström, B., Mayor, M., Andersen, J., et al. 2004, A\&A, 418, 989

Robrade, J., \& Schmitt, J. H. M. M. 2005, A\&A, 435, 1073

Robrade, J., \& Schmitt, J. H. M. M. 2006, A\&A, 449, 737

Sanz-Forcada, J., Maggio, A., \& Micela, G. 2003, A\&A, 408, 1087

Sanz-Forcada, J., Favata, F., \& Micela, G. 2004, A\&A, 416, 281

Savage, B. D., \& Sembach, K. R. 1996, ARA\&A, 34, 279

Scelsi, L., Maggio, A., Peres, G., \& Gondoin, P. 2004, A\&A, 413, 643

Scelsi, L., Maggio, A., Peres, G., \& Pallavicini, R. 2005, A\&A, 432, 671

Schwadron, N. A., Fisk, L. A., \& Zurbuchen, T. H. 1999, ApJ, 521, 859

Sheeley, Jr., N. R. 1996, ApJ, 469, 423

Siess, L., Dufour, E., \& Forestini, M. 2000, A\&A, 358, 593

Smith, K. W., Balega, Y. Y., Duschl, W. J., et al. 2005, A\&A, 431, 307

Stelzer, B., \& Schmitt, J. H. M. M. 2004, A\&A, 418, 687

Strüder, L., Briel, U., Dennerl, K., et al. 2001, A\&A, 365, L18

Telleschi, A., Güdel, M., Briggs, K. R., Audard, M., \& Scelsi, L. 2007, A\&A, 468,443

Telleschi, A., Güdel, M., Briggs, K., et al. 2005, ApJ, 622, 653

Tsuboi, Y., Koyama, K., Murakami, H., et al. 1998, ApJ, 503, 894

Widing, K. G., \& Feldman, U. 1992, ApJ, 392, 715 